\title{
EPR OF Gd ${ }^{3+}$-DOPED La $\mathrm{La}_{0.9} \mathrm{Nd}_{0.1} \mathrm{~F}_{3}$ CRYSTAL: SPIN-PHONON INTERACTIONS AND SPIN-LATTICE RELAXATIONS
}

\author{
M.L. Paradowski* and L.E. Misiak \\ Institute of Physics, Maria Curie-Skłodowska University \\ Pl. M. Curie-Skłodowskiej 1, 20-031 Lublin, Poland
}

(Received June 5, 1998; revised version January 25, 1999)

\begin{abstract}
The $\mathrm{X}$-band EPR study of $\mathrm{Gd}^{3+}$-doped $\mathrm{La}_{0.9} \mathrm{Nd}_{0.1} \mathrm{~F}_{3}$ single crystal in the temperature range $4.2-295 \mathrm{~K}$ is carried out in order to investigate crystal field effects, the $\mathrm{Gd}^{3+}$ spin-phonon interactions, as well as $\mathrm{Gd}^{3+}$ and $\mathrm{Nd}^{3+}$ spin-lattice relaxation times. The local distortions at $4.2 \mathrm{~K}$ of the positions of the eight $\mathrm{F}^{-}$ions surrounding the $\mathrm{Gd}^{3+}$ ion in the $\mathrm{La}_{0.9} \mathrm{Nd}_{0.1} \mathrm{~F}_{3}$ crystal were determined from comparison of the theoretical with the experimental spin-Hamiltonian parameters. The spin-phonon interactions can be described by the Einstein model, which better characterizes the behavior of paramagnetic centers in $\mathrm{LaF}_{3}$ and $\mathrm{La}_{0.9} \mathrm{Nd}_{0.1} \mathrm{~F}_{3}$ crystals than the Debye model. It is suggested, from the rotational invariance mechanism for phonon-induced contributions to spin-Hamiltonian parameters, that the rotational contributions are much smaller than those from the strain.
\end{abstract}

PACS numbers: $76.30 . \mathrm{Kg}$

\section{Introduction}

The $\mathrm{REF}_{3}(\mathrm{RE}=\mathrm{Ce}, \mathrm{Nd})$ crystals are expected to serve as heavy, fast, and radiation hard scintillators for calorimetry at future colliders $[1,2]$. These crystals are also used as laser materials [3, 4].

The electron paramagnetic resonance (EPR) measurements of $\mathrm{Gd}^{3+}$-doped $\mathrm{LaF}_{3}$ and $\mathrm{NdF}_{3}$ single crystals in the temperature range $4.2-295 \mathrm{~K}$ were previously performed by Misra et al. [5,6]. The EPR linewidths were measured in $\mathrm{Gd}^{3+}$-doped $\mathrm{La}_{x} \mathrm{Nd}_{1-x} \mathrm{~F}_{3}(0 \leq x \leq 1)$ single crystals in the temperature range from 77 to $510 \mathrm{~K}[7,8]$, whereas the magnetic susceptibility in the temperature range 1.5-300 K [9]. A temperature EPR (X-band) study of $\mathrm{Gd}^{3+}$-doped $(0.1 \mathrm{~mol} \%) \mathrm{La}_{0.9} \mathrm{Nd}_{0.1} \mathrm{~F}_{3}$ single crystal has been carried out presently in order to

*e-mail: mlpar@tytan.umcs.lublin.pl 
investigate structural and crystal field effects, spin-phonon interactions, and dipolar interactions between $\mathrm{Gd}^{3+}$ and $\mathrm{Nd}^{3+}$ ions. The above sample is the only mixed crystal studied in detail for which well-resolved EPR spectra can be recorded down to liquid-helium temperature.

\section{Sample preparation and crystal structure}

The $\mathrm{La}_{x} \mathrm{Nd}_{1-x} \mathrm{~F}_{3}$ single crystals doped with $\mathrm{Gd}^{3+}(0.1 \mathrm{~mol} \%)$ were grown by a modified Bridgman-Stockbarger method [10]. They were transparent and cleft easily in the cleavage planes (001) and (110).

$\mathrm{LaF}_{3}$ and $\mathrm{NdF}_{3}$ single crystals have a tysonite structure with the trigonal space-group classification $P \overline{3} c 1\left(D_{3 d}^{4}\right.$ trigonal symmetry with a hexamolecular unit cell) $[11,12]$. The site symmetry of the $\mathrm{La}^{3+}$ and the $\mathrm{Nd}^{3+}$ ions is $C_{2}$. The twofold axis is perpendicular to the threefold axis $C_{3}$ in the unit cell. The crystallographic $a$ axis forms an angle of $30^{\circ}$ with respect to one of the three $C_{2}$ axes, where the two remaining $C_{2}$ axes form an angle of $90^{\circ}$ and $150^{\circ}$ with respect to $a$ axis, respectively. The crystallographic $c$ axis is parallel to the $C_{3}$ axis, and perpendicular to the three $C_{2}$ axes. There are six molecules per unit cell. The unit cell parameters for $\mathrm{LaF}_{3}$ are as follows: $a=0.7186 \pm 0.0001 \mathrm{~nm}, c=0.7352 \pm 0.0001 \mathrm{~nm}$, while for $\mathrm{NdF}_{3}$ are: $a=0.7032 \pm 0.0001 \mathrm{~nm}$ and $c=0.7200 \pm 0.0001 \mathrm{~nm}$ at room temperature [10]. Since the values of $a$ and $c$ and the distances of the rare-earth in $\mathrm{LaF}_{3}$ and $\mathrm{NdF}_{3}$ are quite close to each other, it is assumed that $\mathrm{La}_{x} \mathrm{Nd}_{1-x} \mathrm{~F}_{3}$ single crystals also have a tysonite structure, with the $a$ and $c$ dimensions scaled in proportion to $x$ between limit values of lattice constants in $\mathrm{LaF}_{3}$ and $\mathrm{NdF}_{3}$ [13].

\section{Experimental details}

The experimental arrangement has been described elsewhere [14]. The EPR spectra of the $\mathrm{Gd}^{3+}$ ions in $\mathrm{La}_{0.9} \mathrm{Nd}_{0.1} \mathrm{~F}_{3}$ single crystal were recorded with the external magnetic field $\boldsymbol{B}$ oriented in the $Z X$ plane. The $\boldsymbol{Z}(\| \boldsymbol{a})$ axis, along which the overall splitting of EPR lines has a maximum, lies in the cleavage plane (001) and is perpendicular to the (110) plane. The $X$ axis is defined to be the direction of the external magnetic field for which the next-largest extremum occurs. The $\boldsymbol{Y}$ (II $\boldsymbol{c})$ axis is defined to be the direction for which the minimum splitting of EPR lines is observed.

The angular variation for $\mathrm{Gd}^{3+}$-doped $\mathrm{La}_{0.9} \mathrm{Nd}_{0.1} \mathrm{~F}_{3}$ in the $Z X$ plane at $295 \mathrm{~K}$ is shown in Fig. 1. These variations in the $Z X$ plane at room and liquid nitrogen temperatures were found to display three maxima and minima of the three centers placed by $60^{\circ}$ from each other. The six ions in the unit cell were found to be magnetically equivalent in pairs, so that there are three magnetically nonequivalent sites of $\mathrm{Gd}^{3+}$ ions forming three observed centers [15]. Thus, EPR spectra are consistent with the rhombic $C_{2 v}$ site symmetry for the $\mathrm{Gd}^{3+}$ ion in the $D_{3 d}^{4}$ trigonal symmetry with a hexamolecular unit cell. However, only two centers were observed at liquid helium temperature: the $\mathrm{Gd}^{3+}(1)$ center which exhibits the twofold repetition pattern about the $c$ axis ( $Y$ axis) and the $\mathrm{Gd}^{3+}(2)$ center exhibiting the site symmetry close to monoclinic $C_{2}$. It means that the crystal field is changed with lowering the temperature, as a result of the temperature-induced distortion of the crystal lattice [15]. 


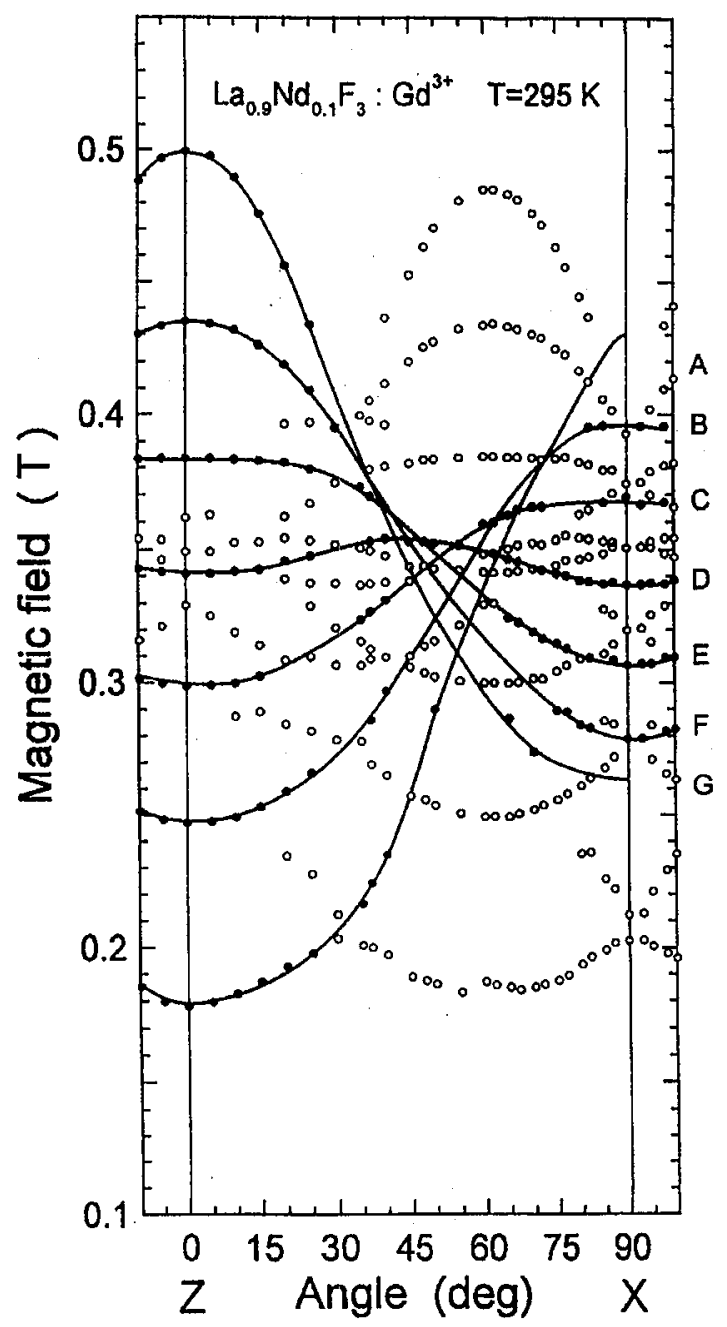

Fig. 1. Angular variation of the EPR lines positions in the $Z X$ plane for $\mathrm{Gd}^{3+}$-doped $\mathrm{La}_{0.9} \mathrm{Nd}_{0.1} \mathrm{~F}_{3}$ single crystal at room temperature. The dots represent the experimental resonant line positions of $\Delta M= \pm 1$ transitions and the solid lines (as a guide for the eye) are smooth curves that connect data points from the same transitions, corresponding to one set (called $\mathrm{Gd}^{3+}(1)$ center) of magnetically equivalent ions. The circles, which represent the experimental line positions of the $\mathrm{Gd}^{3+}(2)$ center, are not connected. The transitions are indicated as follows: $A\left(\frac{7}{2} \leftrightarrow \frac{5}{2}\right), B\left(\frac{5}{2} \leftrightarrow \frac{3}{2}\right), C\left(\frac{3}{2} \leftrightarrow \frac{1}{2}\right)$, $D\left(\frac{1}{2} \leftrightarrow-\frac{1}{2}\right), E\left(-\frac{1}{2} \leftrightarrow-\frac{3}{2}\right), F\left(-\frac{3}{2} \leftrightarrow-\frac{5}{2}\right), G\left(-\frac{5}{2} \leftrightarrow-\frac{7}{2}\right)$.

The average linewidths (peak-to-peak of the first derivative lineshape) $\Delta B_{\mathrm{pp}}$ of the seven EPR lines characterizing the rhombic $C_{2 v}$ site symmetry of $\mathrm{Gd}^{3+}$ in $\mathrm{La}_{0.9} \mathrm{Nd}_{0.1} \mathrm{~F}_{3}$ (for $\boldsymbol{B} \| \boldsymbol{Z}$ ) are equal to $13.8 \pm 0.8 \mathrm{mT}, 10.8 \pm 0.8 \mathrm{mT}, 9.4 \pm 0.6 \mathrm{mT}$, $8.5 \pm 0.6 \mathrm{mT}$, and $8.1 \pm 0.6 \mathrm{mT}$ at $4.2,22,50,77$, and $295 \mathrm{~K}$, respectively. In $\mathrm{LaF}_{3}$ average $\Delta B_{\mathrm{pp}}$ are about $2.5 \pm 0.3 \mathrm{mT}$ in the temperature range $4.2-295 \mathrm{~K}[5]$. 


\section{Spin-Hamiltonian parameters (SHP) and superposition model calculations}

The spin Hamiltonian describing $\mathrm{Gd}^{3+}$ ion in the rhombic $C_{2 v}$ symmetry of the crystal field is given as

$$
\mathcal{H}=\tilde{g} \mu_{\mathrm{B}} \boldsymbol{B} \boldsymbol{S}+\sum_{m=0,2} \frac{1}{3} b_{2}^{m} O_{2}^{m}+\sum_{m=0,2,4} \frac{1}{60} b_{4}^{m} O_{4}^{m}+\sum_{m=0,2,4,6} \frac{1}{1260} b_{6}^{m} O_{6}^{m} .
$$

The symbols have their usual meaning as defined by Abragam and Bleaney [16]. The $S\left(=\frac{7}{2}\right)$ is the electron spin of the $\mathrm{Gd}^{3+}$ ion. The eleven SHP's for $\mathrm{Gd}^{3+}$ in $\mathrm{La}_{0.9} \mathrm{Nd}_{0.1} \mathrm{~F}_{3}$ single crystal were evaluated using a least-squares fitting procedure [17] for fitting simultaneously all resonant line positions recorded in the $Z X$ plane (Table I).

TABLE I

Values of the spin-Hamiltonian parameters in $\mathrm{Gd}^{3+}$-doped $\mathrm{La}_{0.9} \mathrm{Nd}_{0.1} \mathrm{~F}_{3}$ single crystal at various temperatures. The $b_{l}^{m}$ parameters are expressed in $\mathrm{GHz}$, while $g_{z z}$ and $g_{x x}$ are dimensionless; $n$ represents the total number of line positions simultaneously fitted. $S M D\left[\mathrm{GHz}^{2}\right]=\sum_{j}\left(\left|\Delta E_{j}\right|-h \nu_{j}\right)^{2}$, where $\Delta E_{j}$ is the calculated energy difference between levels participating in resonance for the $j$-th resonance magnetic field, and $h \nu_{j}$ is the microwave energy. The coefficient $\alpha$ represents the admixture of state ${ }^{6} P_{7 / 2}$ in the state ${ }^{8} S_{7 / 2}$.

\begin{tabular}{c|c|r|r|r|r}
\hline \hline$T[\mathrm{~K}]$ & \multicolumn{1}{|c|}{295} & \multicolumn{1}{c|}{77} & \multicolumn{1}{c|}{50} & \multicolumn{1}{c}{22} & \multicolumn{1}{c}{4.2} \\
\hline$g_{z z}$ & $1.993 \pm 0.002$ & $1.983 \pm 0.003$ & $1.998 \pm 0.013$ & $1.991 \pm 0.013$ & $1.963 \pm 0.013$ \\
$g_{x x}$ & $1.990 \pm 0.002$ & $1.988 \pm 0.004$ & $1.971 \pm 0.012$ & $1.981 \pm 0.012$ & $1.999 \pm 0.012$ \\
$b_{2}^{0}$ & $0.696 \pm 0.003$ & $0.698 \pm 0.003$ & $0.674 \pm 0.011$ & $0.647 \pm 0.009$ & $0.652 \pm 0.005$ \\
$b_{2}^{2}$ & $-0.121 \pm 0.009$ & $-0.045 \pm 0.014$ & $-0.155 \pm 0.032$ & $-0.167 \pm 0.028$ & $-0.140 \pm 0.021$ \\
$b_{4}^{0}$ & $0.017 \pm 0.001$ & $0.017 \pm 0.001$ & $0.021 \pm 0.004$ & $0.014 \pm 0.003$ & $0.022 \pm 0.001$ \\
$b_{4}^{2}$ & $0.045 \pm 0.007$ & $0.027 \pm 0.009$ & $0.024 \pm 0.015$ & $0.018 \pm 0.012$ & $0.013 \pm 0.012$ \\
$b_{4}^{4}$ & $0.035 \pm 0.011$ & $0.065 \pm 0.015$ & $0.070 \pm 0.031$ & $0.072 \pm 0.025$ & $0.119 \pm 0.019$ \\
$b_{6}^{0}$ & $-0.001 \pm 0.001$ & $-0.001 \pm 0.001$ & $0.003 \pm 0.005$ & $-0.000 \pm 0.003$ & $-0.006 \pm 0.002$ \\
$b_{6}^{2}$ & $0.010 \pm 0.011$ & $0.004 \pm 0.013$ & $0.126 \pm 0.082$ & $-0.049 \pm 0.032$ & $0.006 \pm 0.018$ \\
$b_{6}^{4}$ & $-0.030 \pm 0.018$ & $-0.056 \pm 0.022$ & $-0.080 \pm 0.022$ & $-0.279 \pm 0.029$ & $0.064 \pm 0.029$ \\
$b_{6}^{6}$ & $-0.028 \pm 0.019$ & $-0.009 \pm 0.032$ & $-0.279 \pm 0.109$ & $-0.051 \pm 0.041$ & $-0.101 \pm 0.039$ \\
$n$ & 125 & 94 & 12 & 34 & 65 \\
$S M D$ & 0.208 & 0.151 & 0.098 & 0.127 & 0.684 \\
$\alpha$ & $0.19 \pm 0.02$ & $0.24 \pm 0.02$ & $0.25 \pm 0.09$ & $0.24 \pm 0.06$ & $0.27 \pm 0.03$
\end{tabular}

The crystal-field parameters $b_{l}^{m}$ and the zero-field splittings $\Delta E$ of $\mathrm{Gd}^{3+}$ in $\mathrm{LaF}_{3}$ and $\mathrm{La}_{0.9} \mathrm{Nd}_{0.1} \mathrm{~F}_{3}$ hosts can be fitted in the temperature range $4.2-295 \mathrm{~K}$ to the quadratic function, $a+b T+c T^{2}$, where the values of $a, b, c$ are included in Table II. A quadratic temperature dependence of $b_{l}^{m}$ was observed for $\mathrm{Gd}^{3+}$ in $\mathrm{LiY}_{1-x} \mathrm{Yb}_{x} \mathrm{~F}_{4}$ hosts $[14,18]$, assuming the temperature dependence is due to static distortion. On the other hand, vibrational effects of the crystal lattice cause temperature dependence of $b_{l}^{m}$ which can be fitted to the equation containing terms $\sum_{i} d_{i} \operatorname{coth}\left(\frac{h \nu_{i}}{2 k T}\right)$, where $\nu_{i}$ are the lattice vibration frequencies and $d_{i}$ are 
TABLE II

Values of the constants required to fit the spin-Hamiltonian parameters and the zero-field splitting: $\left(b_{l}^{m}\right.$ and $\left.\Delta E\right)=a+b T+c T^{2}$ in the temperature range $4.2-295 \mathrm{~K}$. The $\chi^{2}$ values characterizing these fits are also included.

\begin{tabular}{c|c|c|r|r|c}
\hline \hline \multicolumn{2}{c|}{ Sample } & \multicolumn{1}{c|}{$a[\mathrm{GHz}]$} & \multicolumn{1}{c}{$b\left[\mathrm{GHz} \cdot \mathrm{K}^{-1}\right]$} & \multicolumn{1}{c}{$c[\mathrm{GHz} \cdot \mathrm{K}-2]$} & $\chi^{2}\left[\mathrm{GHz}^{2}\right]$ \\
\hline \multirow{3}{*}{$\mathrm{LaF}_{3}$} & $b_{2}^{0}$ & 0.7015 & $0.6310 \times 10^{-5}$ & $-0.1042 \times 10^{-6}$ & $5.2362 \times 10^{-5}$ \\
& $b_{2}^{2}$ & $-0.1110 \times 10^{-1}$ & $-0.5737 \times 10^{-3}$ & $0.1132 \times 10^{-5}$ & $2.2955 \times 10^{-4}$ \\
& $b_{4}^{0}$ & $0.1885 \times 10^{-1}$ & $-0.6496 \times 10^{-4}$ & $0.1902 \times 10^{-6}$ & $2.6879 \times 10^{-6}$ \\
& $b_{4}^{2}$ & $0.5383 \times 10^{-1}$ & $0.1284 \times 10^{-3}$ & $-0.3059 \times 10^{-6}$ & $1.5557 \times 10^{-4}$ \\
& $b_{4}^{4}$ & 0.1212 & $-0.3751 \times 10^{-3}$ & $0.1211 \times 10^{-5}$ & $9.0903 \times 10^{-6}$ \\
& $\Delta E$ & 8.3503 & $0.1286 \times 10^{-2}$ & $-0.3955 \times 10^{-5}$ & $3.2892 \times 10^{-3}$ \\
\hline \multirow{4}{*}{$\mathrm{La}_{0.9} \mathrm{Nd}_{0.1} \mathrm{~F}_{3}$} & $b_{2}^{0}$ & 0.6412 & $0.8183 \times 10^{-3}$ & $-0.2146 \times 10^{-5}$ & $2.2482 \times 10^{-4}$ \\
& $b_{2}^{2}$ & -0.1750 & $0.1516 \times 10^{-2}$ & $-0.4508 \times 10^{-5}$ & $4.9005 \times 10^{-3}$ \\
& $b_{4}^{0}$ & $0.1894 \times 10^{-1}$ & $-0.8995 \times 10^{-5}$ & $7.6012 \times 10^{-8}$ & $4.1983 \times 10^{-5}$ \\
& $b_{4}^{2}$ & $0.1434 \times 10^{-1}$ & $0.1713 \times 10^{-3}$ & $-0.2285 \times 10^{-6}$ & $8.5805 \times 10^{-6}$ \\
& $b_{4}^{4}$ & 0.1063 & $-0.7255 \times 10^{-3}$ & $0.1658 \times 10^{-5}$ & $6.3936 \times 10^{-4}$ \\
& $\Delta E$ & 7.8387 & $0.8402 \times 10^{-2}$ & $-0.2156 \times 10^{-4}$ & $5.9427 \times 10^{-3}$
\end{tabular}

the weighting parameters [19]. These terms in temperature expansion of $b_{l}^{m}(T)$ will then contain terms in $T, T^{2}$, and higher powers.

The SHP's for $\mathrm{Gd}^{3+}{ }_{\text {-doped }} \mathrm{La}_{0.9} \mathrm{Nd}_{0.1} \mathrm{~F}_{3}$ single crystal can be analyzed using the superposition model [20]. In this model the $b_{l}^{m}$ are expressed as linear superpositions of single-ligand contributions to the intrinsic parameters $\vec{b}_{l}\left(R_{0}\right)$ which depend on the ligand $i$ as follows:

$$
b_{l}^{m}=\sum_{i} \bar{b}_{l}\left(R_{i}\right) K_{l}^{m}\left(\theta_{i}, \varphi_{i}\right),
$$

where

$$
\bar{b}_{l}\left(R_{i}\right)=\bar{b}_{l}\left(R_{0}\right)\left(R_{0} / R_{i}\right)^{t_{l}}
$$

The $R_{i}$ 's in Eqs. (2) and (3) are the distances of the ligand $\mathrm{F}^{-}$ions from the $\mathrm{Gd}^{3+}$ ion, $\theta_{i}$ is the polar angle of the $i$-th $\mathrm{F}^{-}$ion with respect to the $Z$ axis, $\varphi_{i}$ is the azimuthal angle of the $i$-th $\mathrm{F}^{-}$ion with respect to the $X$ axis and the $R_{0}$ is a particular bond length, used as a reference (i.e. the minimum distance $\mathrm{F}^{-}-\mathrm{Gd}^{3+}$ ). The $K_{l}^{m}$ in Eq. (2) are angular functions related to the spherical harmonics [21].

For the $\mathrm{La}_{x} \mathrm{Nd}_{1-x} \mathrm{~F}_{3}$ single crystals $(x=1,0.9,0.1)$, we consider the nine nearest neighbor $\mathrm{F}^{-}$ions to a $\mathrm{Gd}^{3+}$ ion which substitute for $\mathrm{La}^{3+}$, or $\mathrm{Nd}^{3+}$ ion (Fig. 2). The required positions $\left(R_{i}, \theta_{i}, \varphi_{i}\right)$ for $\mathrm{LaF}_{3}$ and $\mathrm{NdF}_{3}$ are given in Ref. [6]. In order to evaluate the intrinsic parameters $\bar{b}_{l}\left(R_{0}\right)$, the required lattice constants of $\mathrm{La}_{0.9} \mathrm{Nd}_{0.1} \mathrm{~F}_{3}$ were estimated using Vegard's law [13]. The unit cell parameters of $\mathrm{LaF}_{3}$ and $\mathrm{NdF}_{3}$ were measured in the range 87-295 $\mathrm{K}$ [10]; those parameters at lower temperatures were obtained by extrapolation. The application of the superposition model requires exact knowledge of $t_{l}$ intrinsic exponential parameters and positions of ligands around the paramagnetic ion. In our case we assumed that $t_{2}=9$ and $t_{4}=14$, which are the same as those for $\mathrm{LaF}_{3}$ and $\mathrm{NdF}_{3}$ hosts [6]. The angular coordinations of $\mathrm{F}^{-}$ligands were changed independently in the calculations from Ref. [6]. We propose to change the angles 


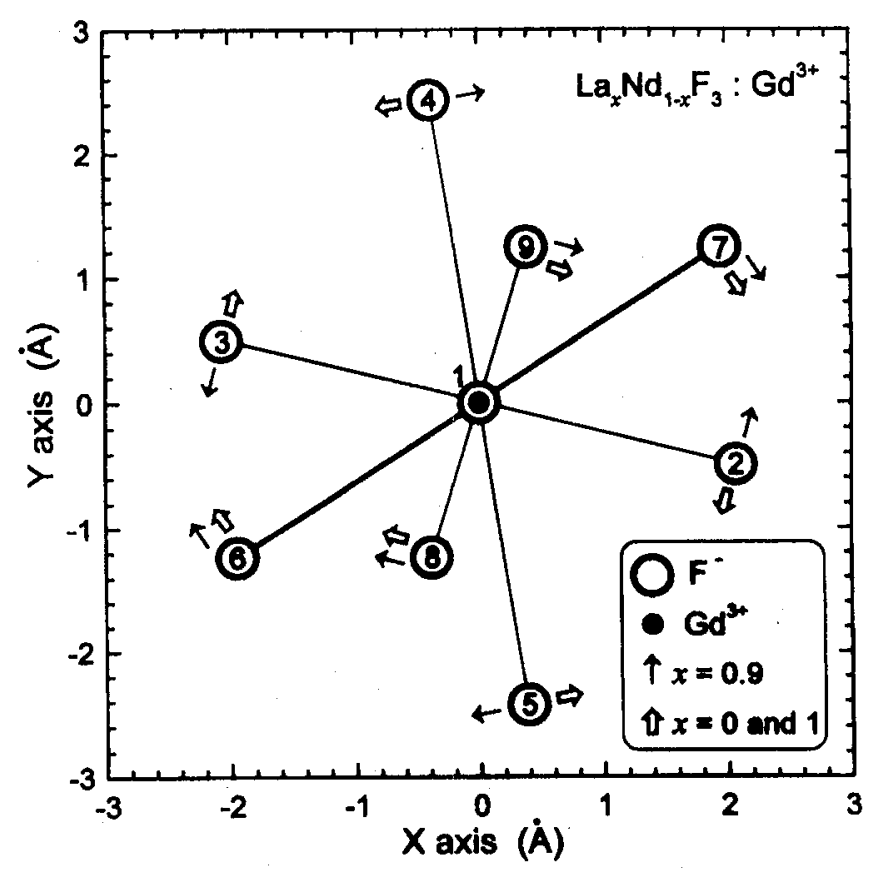

Fig. 2. The projection of $\mathrm{F}^{-}$ions onto the $X Y$ plane showing the deformation of the $\mathrm{La}_{x} \mathrm{Nd}_{1-x} \mathrm{~F}_{3}$ host lattice at room temperature after the $\mathrm{Gd}^{3+}$ ion substitute for the $\mathrm{La}^{3+}$ or the $\mathrm{Nd}^{3+}$ ion. The fluorine ion 1 is above the plane and sets the $Z$ axis. The fluorine ions 6 and 7 lie above the $X Y$ plane (connection to the $\mathrm{Gd}^{3+}$ ion marked by solid thick lines), whereas remaining fluorides lie below this plane (thin lines).

$\varphi$ of $\mathrm{F}^{-}$pairs at the coordinates $(R, \theta, \varphi)$ and $\left(R, \theta, \varphi+180^{\circ}\right)$ by the same values. In our calculations for $\mathrm{Gd}^{3+}{ }_{- \text {doped }} \mathrm{LaF}_{3}, \mathrm{La}_{0.9} \mathrm{Nd}_{0.1} \mathrm{~F}_{3}$ and $\mathrm{NdF}_{3}$ we have attempted a direct fitting to the experimental parameters $b_{l}^{m}$, allowing the angular azimuthal coordinates for eight $\mathrm{F}^{-}$ligands (grouped in pairs) to vary within $\Delta \varphi= \pm 3.5^{\circ}\left(\Delta \varphi=\varphi_{\text {final }}-\varphi_{\text {initial }}\right)$. The deformation (rotation) is around the $Z$-axis; i.e. the $\mathrm{F}^{-}$ions rotate in the $X Y$ plane perpendicular to the crystallographic $a$ axis, according to arrows in Fig. 2. Combining Eqs. (2) with (3) we were able to compute angles of local distortions $(\Delta \varphi)$ of eight ligands grouped in pairs, intrinsic parameters $\bar{b}_{2}$ and $\bar{b}_{4}$, and theoretical values of $b_{l}^{m}$ at various temperatures (Table III). There is a good agreement of the calculated theoretical value with the experimental values of the $b_{l}^{m}$, supporting the above mechanism of distortion. There are local distortions (rotations) in opposite directions $\Delta \varphi$ for 2-3 and 4-5 $\mathrm{F}^{-}$pairs in $\mathrm{La}_{0.9} \mathrm{Nd}_{0.1} \mathrm{~F}_{3}$ (Fig. 2), contrary to those in $\mathrm{LaF}_{3}$ and $\mathrm{NdF}_{3}$ at room temperature, where only $4-5 \mathrm{~F}^{-}$pairs are rotated in direction opposite to other $\mathrm{F}^{-}$pairs. The rotation changes to opposite directions for $4-5$ and $8-9$ $\mathrm{F}^{-}$pairs are also observed in $\mathrm{La}_{0.9} \mathrm{Nd}_{0.1} \mathrm{~F}_{3}$ when temperature is lowering down to $4.2 \mathrm{~K}$ (see Table III). This confirms our earlier suggestion [15] that the crystal field in $\mathrm{La}_{0.9} \mathrm{Nd}_{0.1} \mathrm{~F}_{3}$ is changed with lowering temperature, as a result of temperature 
TABLE III

The experimental (Exp.) and theoretical calculated from superposition model (Theo.) values of spin-Hamiltonian parameters $b_{l}^{m}$ (in $\mathrm{GHz}$ ). The intrinsic parameters $\bar{b}_{l}$ (in $\mathrm{GHz}$ ) and the local distortion measured by azimuthal angles $\Delta \varphi_{i, j}$ (in degrees) from Gd ${ }^{3+}$ ion to eight surrounding $\mathrm{F}^{-}$ions are also included; $R_{0}$ is the minimum distance between $\mathrm{Gd}^{3+}-\mathrm{F}^{-}$ions. $\Delta E=E\left( \pm \frac{7}{2}\right)-E\left( \pm \frac{1}{2}\right)$ (in $\mathrm{GHz}$ ) is the zero-field splitting.

\begin{tabular}{|c|c|c|c|c|c|c|c|c|c|c|c|c|c|c|}
\hline \multirow{3}{*}{$\frac{\text { Sample }}{T[\mathrm{~K}]}$} & \multirow{2}{*}{\multicolumn{2}{|c|}{$\frac{\mathrm{LaF}_{3}}{295}$}} & \multicolumn{10}{|c|}{$\mathrm{La}_{0.9} \mathrm{Nd}_{0.1} \mathrm{~F}_{3}$} & \multirow{2}{*}{\multicolumn{2}{|c|}{$\frac{\mathrm{NdF}_{3}}{295}$}} \\
\hline & & & \multicolumn{2}{|c|}{295} & \multicolumn{2}{|c|}{77} & \multicolumn{2}{|c|}{50} & \multicolumn{2}{|c|}{22} & \multicolumn{2}{|c|}{4.2} & & \\
\hline & Exp. $^{a}$ & Theo. & Exp. & Theo. & Exp. & Theo. & Exp. & Theo. & Exp. & Theo. & Exp. & Theo. & Exp. $^{a}$ & Theo. \\
\hline$b_{2}^{0}$ & 0.694 & 0.707 & 0.696 & 0.691 & 0.698 & 0.697 & 0.674 & 0.677 & 0.647 & 0.648 & 0.652 & 0.654 & 0.802 & 0.809 \\
\hline$b_{2}^{2}$ & -0.083 & -0.082 & -0.121 & -0.122 & -0.045 & -0.045 & -0.155 & -0.154 & -0.167 & -0.167 & -0.140 & -0.140 & -0.148 & -0.147 \\
\hline$b_{4}^{0}$ & 0.016 & 0.016 & 0.017 & 0.014 & 0.017 & 0.014 & 0.021 & 0.016 & 0.014 & 0.011 & 0.022 & 0.016 & 0.019 & 0.019 \\
\hline$b_{4}^{2}$ & 0.066 & 0.072 & 0.045 & 0.053 & 0.027 & 0.057 & 0.024 & 0.064 & 0.018 & 0.046 & 0.013 & 0.071 & 0.081 & 0.084 \\
\hline$b_{4}^{4}$ & 0.117 & 0.108 & 0.035 & 0.038 & 0.065 & 0.052 & 0.070 & 0.053 & 0.072 & 0.056 & 0.119 & 0.084 & 0.130 & 0.127 \\
\hline $\bar{b}_{2}$ & \multicolumn{2}{|c|}{$-4.616 \pm 0.050$} & \multicolumn{2}{|c|}{$-4.547 \pm 0.046$} & \multicolumn{2}{|c|}{$-4.622 \pm 0.080$} & \multicolumn{2}{|c|}{$-4.470 \pm 0.096$} & \multicolumn{2}{|c|}{$-4.250 \pm 0.089$} & \multicolumn{2}{|c|}{$-4.273 \pm 0.081$} & \multicolumn{2}{|c|}{$-5.497 \pm 0.050$} \\
\hline $\bar{b}_{4}$ & \multicolumn{2}{|c|}{$0.018 \pm 0.001$} & \multicolumn{2}{|c|}{$0.015 \pm 0.003$} & \multicolumn{2}{|c|}{$0.015 \pm 0.002$} & \multicolumn{2}{|c|}{$0.018 \pm 0.008$} & \multicolumn{2}{|c|}{$0.012 \pm 0.005$} & \multicolumn{2}{|c|}{$0.018 \pm 0.002$} & \multicolumn{2}{|c|}{$0.021 \pm 0.001$} \\
\hline$\Delta \varphi_{2,3}$ & \multicolumn{2}{|c|}{$2.0 \pm 0.5$} & \multicolumn{2}{|c|}{$-3.5 \pm 0.5$} & \multicolumn{2}{|c|}{$-3.5 \pm 0.5$} & \multicolumn{2}{|c|}{$-3.5 \pm 0.5$} & \multicolumn{2}{|c|}{$0.0 \pm 0.5$} & \multicolumn{2}{|c|}{$-3.5 \pm 0.5$} & \multicolumn{2}{|c|}{$2.0 \pm 0.5$} \\
\hline$\Delta \varphi_{4,5}$ & \multicolumn{2}{|c|}{$-3.5 \pm 0.5$} & \multicolumn{2}{|c|}{$3.5 \pm 0.5$} & \multicolumn{2}{|c|}{$1.0 \pm 0.5$} & \multicolumn{2}{|c|}{$2.5 \pm 0.5$} & $0.0=$ & $=0.5$ & $-3.0=$ & $=0.5$ & $-3.5=$ & 0.5 \\
\hline$\Delta \varphi_{6,7}$ & 3.5 & $=0.5$ & $3.0=$ & $=0.5$ & $2.5=$ & $=0.5$ & $2.5=$ & 0.5 & $3.5=$ & $=0.5$ & $1.0=$ & 0.5 & $3.5=$ & 0.5 \\
\hline$\Delta \varphi_{8,9}$ & 2.0 & $=0.5$ & $3.0=$ & $=0.5$ & $-1.0=$ & $=0.5$ & $2.0=$ & 0.5 & $0.5=$ & $=0.5$ & $-3.5=$ & $=0.5$ & $3.0=$ & 0.5 \\
\hline$R_{0}[\AA]$ & 2.4 & 187 & 2.4 & 133 & 2.4 & & 2.4 & 990 & 2.4 & 092 & 2.4 & & 2.3 & \\
\hline$\Delta E$ & $8.39=$ & $0.10^{a}$ & $8.44=$ & $=0.04$ & $8.36 \pm$ & $=0.05$ & $8.23=$ & $=0.20$ & $7.95=$ & $=0.15$ & $7.91 \pm$ & 0.08 & $9.80 \pm$ & $0.10^{a}$ \\
\hline
\end{tabular}

${ }^{a}$ The experimental SHP's were taken from Refs. [6] and P. Mikołajczak, Hab. Thesis, Maria Curie-Skłodowska University, Lublin 1982. 
induced distortion of crystal lattice which causes the change of the site symmetry of $\mathrm{Gd}^{3+}$ ion from $C_{2 v}$ at $77 \mathrm{~K}$ towards close $C_{2}$ at $4.2 \mathrm{~K}$. In $\mathrm{LaF}_{3}$ any rotation changes to opposite directions are not observed with lowering temperature down to $4.2 \mathrm{~K}$. The distortions of four fluorine pairs in the same directions as at $295 \mathrm{~K}$ only remain.

\section{Debye and Einstein models of phonon spectrum}

The major part of the temperature dependence of $b_{2}^{0}$ is due to the spin-phonon interaction effects [14]. The $b_{2}^{0}$ parameter changes its value as much as $\Delta b_{2}^{0}=$ $0.0073 \mathrm{GHz}(100 \%)$ in the temperature range $87-295 \mathrm{~K}_{\text {for }} \mathrm{LaF}_{3}$. Using the data of lattice constants dependent on temperature [10] and the equation for $\bar{b}_{2}$ obtained from Eqs. (2), (3) we evaluate to $0.0024 \mathrm{GHz}$ (about $33 \%$ ) the contribution of thermal expansion to $\Delta b_{2}^{0}$. The remaining part (67\%) of the $b_{2}^{0}$ change with temperature is due to the modulation of the crystal field by thermally excited phonons. Thus, the temperature dependence of the $b_{2}^{0}$ can be ascribed to the spin-phonon interaction. The Debye model in the long-wavelength limit gives [22, 23]:

$$
b_{2}^{0}(T)=b_{2}^{0}(0)\left(1-C \frac{T^{4}}{\theta_{\mathrm{D}}^{4}} \int_{0}^{\theta_{\mathrm{D}} / T} \frac{x^{3}}{\mathrm{e}^{x}-1} \mathrm{~d} x\right),
$$

where $C$ is a constant and $\theta_{\mathrm{D}}$ is the Debye temperature. On the other hand, the Einstein model involving a single oscillator gives

$$
b_{2}^{0}(T)=b_{2}^{0}(0)\left\{1-C\left[\operatorname{coth}\left(\frac{h \nu}{2 k T}\right)-1\right]\right\},
$$

where $h \nu$ is energy of the interacting phonon mode. The parameters for the best fit of the $b_{2}^{0}$ to temperature in $\mathrm{La}_{0.9} \mathrm{Nd}_{0.1} \mathrm{~F}_{3}\left(\mathrm{LaF}_{3}\right)$ for the Debye model are

$$
\begin{aligned}
& b_{2}^{0}(0)=0.653 \pm 0.063 \mathrm{GHz}(0.703 \pm 0.070 \mathrm{GHz}), \\
& C=0.110 \pm 0.011(-0.003 \pm 0.005), \\
& \theta_{\mathrm{D}}^{\prime}=224 \pm 12 \mathrm{~K}(218 \pm 8 \mathrm{~K}), \\
& \chi^{2}=1.05 \times 10^{-4} \mathrm{GHz}^{2}\left(1.75 \times 10^{-4} \mathrm{GHz}^{2}\right) .
\end{aligned}
$$

On the other hand, the parameters providing the best fit using the Einstein model are

$$
\begin{aligned}
& b_{2}^{0}(0)=0.668 \pm 0.067 \mathrm{GHz}(0.704 \pm 0.052 \mathrm{GHz}), \\
& C=-0.071 \pm 0.008(0.022 \pm 0.008), \\
& h \nu / k=434 \pm 16 \mathrm{~K}(406 \pm 15 \mathrm{~K}), \\
& \chi^{2}=1.58 \times 10^{-3} \mathrm{GHz}^{2}\left(1.01 \times 10^{-4} \mathrm{GHz}^{2}\right) .
\end{aligned}
$$

There is a good agreement between the $b_{2}^{0}(0)$ values of both models for $\mathrm{La}_{0.9} \mathrm{Nd}_{0.1} \mathrm{~F}_{3}$ and $\mathrm{LaF}_{3}$ single crystals, confirming the correctness of the fitting. The effective Debye temperatures $\theta_{\mathrm{D}}^{\prime}$ for $\mathrm{La}_{0.9} \mathrm{Nd}_{0.1} \mathrm{~F}_{3}$ and $\mathrm{LaF}_{3}$, equal to $224 \mathrm{~K}$ and $218 \mathrm{~K}$, respectively, seem to be too low and it is concluded that the errors originate in the use of the Debye model. The energies of single Einstein oscillators $h \nu / k$ 
used in Eq. (5) are equal to $434 \mathrm{~K}$ and $406 \mathrm{~K}$ for $\mathrm{La}_{0.9} \mathrm{Nd}_{0.1} \mathrm{~F}_{3}\left(\mathrm{Gd}^{3+}\right)$ and $\mathrm{LaF}_{3}$ $\left(\mathrm{Gd}^{3+}\right)$ respectively, and their values are more close to those $382 \mathrm{~K}$ and $387 \mathrm{~K}$ reported for $\mathrm{LaF}_{3}$ and $\mathrm{NdF}_{3}$ in Ref. [24]. The ratio of $\theta_{\mathrm{D}}^{\prime}$ to $h \nu / k$ is equal to 0.5 for both samples and means that the Einstein model yields an interaction with somewhat higher-frequency phonons than the Debye model. The ratio is different from that equal to 1.4 determined in $\mathrm{LiYF}_{4}[25]$ and $\mathrm{BaTiO}_{3}$ [23]. It is clear that in $\mathrm{La}_{x} \mathrm{Nd}_{1-x} \mathrm{~F}_{3}\left(\mathrm{Gd}^{3+}\right)$ system are produced higher frequency phonon modes than in $\mathrm{LiYF}_{4}\left(\mathrm{Gd}^{3+}\right)$ and $\mathrm{BaTiO}_{3}\left(\mathrm{Cr}^{3+}\right)$, presumably due to higher difference of masses of composite ions, as well as to more simple system than in case of $\mathrm{LiYF}_{4}$ and $\mathrm{BaTiO}_{3}$. It is concluded that average phonon spectrum shifts to somewhat shorter wavelength values in $\mathrm{La}_{x} \mathrm{Nd}_{1-x} \mathrm{~F}_{3}\left(\mathrm{Gd}^{3+}\right)(x=1,0.9)$ than long-wavelength limit described by the Debye model (Eq. (4)). The Einstein model of single oscillator better describes the paramagnetic center behavior, i.e., the temperature dependence of $b_{2}^{0}$ parameter, particularly in the case of the presence of one kind of paramagnetic ions (for $\mathrm{Gd}^{3+}$-doped $\mathrm{LaF}_{3}$ crystal). Further, the average energy of thermally excited optical phonons is presently determined to be $282 \mathrm{~cm}^{-1}$ for $\mathrm{Gd}^{3+}$-doped $\mathrm{LaF}_{3}$ and $302 \mathrm{~cm}^{-1}$ for $\mathrm{Gd}^{3+}$-doped $\mathrm{La}_{0.9} \mathrm{Nd}_{0.1} \mathrm{~F}_{3}$. The optical phonons of $E_{\mathrm{g}}$ symmetry were observed at 293, 299.8 and $315.1 \mathrm{~cm}^{-1}$ in $\mathrm{LaF}_{3}$ [26]. The Stokes vibronic emission lines in $\mathrm{Gd}^{3+}{ }_{\text {-doped }} \mathrm{LaF}_{3}$ at $4.2 \mathrm{~K}$ are reported to be 179,289 , 318,362 , and $433 \mathrm{~cm}^{-1}$ [27]. Further, in Raman spectra of $\mathrm{LaF}_{3}$ and $\mathrm{NdF}_{3}$ were observed strong "phonon $E_{\mathrm{g}}$ type" bands at $290 \mathrm{~cm}^{-1}$ in $\mathrm{LaF}_{3}$, and at $314 \mathrm{~cm}^{-1}$ in $\mathrm{NdF}_{3}$ [28]. Taking into account the above comparison, our estimation of optical phonons energy from EPR data is in sufficiently good agreement with those given in the literature.

The average velocity of sound $v_{\mathrm{m}}$ can be estimated from the relation [29]:

$$
v_{m}=\frac{\theta_{\mathrm{D}}}{\frac{h}{k}\left(\frac{3 q}{4 \pi} \frac{N \rho}{M}\right)^{1 / 3}},
$$

where $\rho$ is the density of $\mathrm{LaF}_{3}\left(5.94 \mathrm{~g} \mathrm{~cm}^{-3}\right)$ or $\mathrm{La}_{0.9} \mathrm{Nd}_{0.1} \mathrm{~F}_{3}\left(6.16 \mathrm{~g} \mathrm{~cm}^{-3}\right), q$ is the number of atoms in the molecule ( 4 in these crystals), $N$ is the Avogadro number and $M$ is the molar mass.

The calculated velocities of sound $v_{\mathrm{m}}$ from Eq. (6), using $\theta_{\mathrm{D}}(=h \nu / k)$ equal to $406 \mathrm{~K}$ for $\mathrm{LaF}_{3}$ and to $434 \mathrm{~K}$ for $\mathrm{La}_{0.9} \mathrm{Nd}_{0.1} \mathrm{~F}_{3}$, are $3.26 \times 10^{5} \mathrm{~cm} \mathrm{~s}^{-1}$ and $3.45 \times 10^{5} \mathrm{~cm} \mathrm{~s}^{-1}$, respectively. The velocity of sound in the $X Y$ plane of $\mathrm{LaF}_{3}$ at room temperature was calculated in Ref. [24] as equal to $2.78 \times 10^{5} \mathrm{~cm} \mathrm{~s}^{-1}$. Schulz and Jeffries [30] measured the velocity of sound in $\mathrm{LaF}_{3}$ single crystal and received in average $3.44 \times 10^{5} \mathrm{~cm} \mathrm{~s}^{-1}$. Our calculated $v_{\mathrm{m}}$ 's are quite close to that of measured by Schulz and Jeffries [30] supporting the value of $\theta_{\mathrm{D}}=406 \mathrm{~K}$ in $\mathrm{LaF}_{3}$ to be more realistic than $382 \mathrm{~K}$ calculated in Ref. [24].

Below we consider the successful use of intrinsic parameters to describe the spin-phonon interactions in rare-earth trifluorides. The temperature dependence of $b_{2}^{0}$ value is reflected by the change in the value of the intrinsic parameter $\bar{b}_{2}$ with temperature, because the effect of thermal expansion (contraction) of the lattice on $\bar{b}_{2}$ is calculated to be rather small. In order to describe the temperature dependence of $\bar{b}_{2}$ we used the transformed isotropic Einstein model for lattice 
vibration [31], which contains the coupling between $\mathrm{Gd}^{3+}$ spin and one localized mode

$$
\bar{b}_{2}(T)=\bar{b}_{2}(\mathrm{RL})+K_{2} \operatorname{coth}\left(\frac{h \nu}{2 k T}\right)
$$

where $\bar{b}_{2}(\mathrm{RL})$ is a "rigid lattice" value of $\vec{b}_{2}$ (i.e. the value of $\bar{b}_{2}$ at $T=0 \mathrm{~K}$ minus zero point vibrations) and $K_{2}$ is the coupling constant describing the magnitude of $\mathrm{Gd}^{3+}$ spin-phonon interaction. The $\bar{b}_{2}(\mathrm{RL}), K_{2}$ estimated from the fitting to Eq. (7) yield values $-4.75 \pm 0.05,0.13 \pm 0.02 \mathrm{GHz}$ for $\mathrm{La}_{0.9} \mathrm{Nd}_{0.1} \mathrm{~F}_{3}$ and $-4.85 \pm 0.05,0.14 \pm 0.02 \mathrm{GHz}$ for $\mathrm{LaF}_{3}$, respectively.

On the other hand, the Debye model assumes that paramagnetic ions $\left(\mathrm{Gd}^{3+}\right)$ are coupled to the whole phonon spectrum of the crystal lattice. The equation adopted to fit $\bar{b}_{2}$ to temperature is the following [32]:

$$
\bar{b}_{2}(T)=\bar{b}_{2}(\mathrm{RL})+K_{2}\left(1+8 \frac{T^{4}}{\theta_{\mathrm{D}}^{4}} \int_{0}^{\theta_{\mathrm{D}} / T} \frac{x^{3}}{\mathrm{e}^{x}-1} \mathrm{~d} x\right) .
$$

The best fit with $\theta_{\mathrm{D}}=434 \mathrm{~K}$ and $406 \mathrm{~K}$ for $\mathrm{La}_{0.9} \mathrm{Nd}_{0.1} \mathrm{~F}_{3}$ and $\mathrm{LaF}_{3}$, respectively, yields the following parameters:

$$
\bar{b}_{2}(\mathrm{RL})=-4.71 \pm 0.06 \mathrm{GHz}, \quad K_{2}=0.08 \pm 0.04 \mathrm{GHz}\left(\mathrm{La}_{0.9} \mathrm{Nd}_{0.1} \mathrm{~F}_{3}\right)
$$

and

$$
\bar{b}_{2}(\mathrm{RL})=-4.81 \pm 0.05 \mathrm{GHz}, \quad K_{2}=0.09 \pm 0.03 \mathrm{GHz}\left(\mathrm{LaF}_{3}\right) .
$$

The above $\bar{b}_{2}$ (RL) and $K_{2}$ values derived from the Einstein and the Debye models are equal to each other within errors, confirming the correctness of the fitting.

The coupling between spins and phonons $\left(K_{2}\right)$ in $\mathrm{LaF}_{3}\left(\mathrm{Gd}^{3+}\right)$ is about four times greater than in $\mathrm{LiYF}_{4}\left(\mathrm{Gd}^{3+}\right)$ crystal [18]. This statement can be confirmed by optical measurements [33], implying a larger amplitude of zero vibrations of the lattice in $\mathrm{LaF}_{3}\left(469 \times 10^{-3} \mathrm{~nm}\right)$ versus $\mathrm{LiYF}_{4}\left(3.77 \times 10^{-3} \mathrm{~nm}\right)$.

\section{Rotational invariance and the phonon-induced contributions to SHP}

The theory of rotational invariance for the phonon-induced contributions to spin-Hamiltonian parameters developed by Bates and Szymczak [34, 35] gives more precise description of the observed contribution to the $b_{2}^{0}$ from lattice dynamics. It is usually difficult to determine magnetoelastic tensor components in low symmetry crystal field, e.g., in $\mathrm{REF}_{3}$ crystals. In order to explain experimentally measured SHP's by the above mechanism we need to separate the thermal expansion from that of the spin-phonon contributions. In order to separate the spin-phonon contribution we used the $\bar{b}_{2}$ values determined from superposition model as follows: $\delta \mathcal{D} \approx 0.67 \cdot \delta \bar{b}_{2}=0.67\left[\bar{b}_{2}(295)-\bar{b}_{2}(\mathrm{RL})\right]$. (In Sec. 5 we evaluated to $67 \%$ the spin-phonon contribution, the rest part is the contribution from thermal expansion.) Thus, these spin-phonon contributions $(\delta \mathcal{D})$ are equal to $0.106 \pm 0.036 \mathrm{GHz}$ and $0.129 \pm 0.034 \mathrm{GHz}$ for $\mathrm{Gd}^{3+}+$ doped $\mathrm{La}_{0.9} \mathrm{Nd}_{0.1} \mathrm{~F}_{3}$ and $\mathrm{LaF}_{3}$, respectively.

Further, the anharmonic contributions $\delta \mathcal{D}$ and $\delta \mathcal{D}_{\mathrm{r}}$ to $\mathcal{D}$ are given by the equations [35]:

$$
\delta \mathcal{D}=\frac{-\hbar}{32 \pi^{2} \rho}\left(G_{11}+G_{12}+G_{13}\right)\left(v_{1}^{-5}+2 v_{\mathrm{t}}^{-5}\right) f\left(\omega_{\mathrm{D}}, T\right)
$$


and

$$
\delta \mathcal{D}_{\mathrm{r}}=\frac{-\hbar}{32 \pi^{2} \rho} \mathcal{D} v_{\mathrm{t}}^{-5} f\left(\omega_{\mathrm{D}}, T\right)
$$

where $G_{11}, G_{12}, G_{13}$ are the components of the magnetoelastic tensor, $v_{1}$ and $v_{\mathrm{t}}$ are longitudinal and transversal velocities of sound, respectively, and

$$
f\left(\omega_{\mathrm{D}}, T\right)=\omega_{\mathrm{D}}^{4}+8\left(\frac{k T}{\hbar}\right)^{4} \int_{0}^{\theta_{\mathrm{D}} / T} \frac{x^{3}}{\mathrm{e}^{x}-1} \mathrm{~d} x
$$

with $\hbar \omega_{\mathrm{D}}=k \theta_{\mathrm{D}}$.

The values of sound velocities are $v_{1}=6.02 \times 10^{5} \mathrm{~cm} \mathrm{~s}^{-1}, v_{\mathrm{t}}=$ $2.31 \times 10^{5} \mathrm{~cm} \mathrm{~s}^{-1}$ for $\mathrm{La}_{0.9} \mathrm{Ndd}_{0.1} \mathrm{~F}_{3}$ and $v_{1}=5.69 \times 10^{5} \mathrm{~cm} \mathrm{~s} \mathrm{~s}^{-1}, v_{\mathrm{t}}=$ $2.18 \times 10^{5} \mathrm{~cm} \mathrm{~s}^{-1}$ for $\mathrm{LaF}_{3}$.

The calculation yields the sum values of magnetoelastic tensor components $\left(G_{11}+G_{12}+G_{13}\right)$ to be $-3.04 \pm 1.00$ and $-3.33 \pm 0.88 \mathrm{GHz}$ for $\mathrm{La}_{0.9} \mathrm{Nd}_{0.1} \mathrm{~F}_{3}$ and $\mathrm{LaF}_{3}$, respectively. Further, from Eq. (10) $\delta \mathcal{D}_{\mathrm{r}}$ was determined to be $-0.0121 \pm$ 0.0014 and $-0.0134 \pm 0.0017 \mathrm{GHz}$, as well as the ratio $\mathcal{R}_{\mathrm{D}}\left(=\delta \mathcal{D}_{\mathrm{r}} / \delta \mathcal{D}\right)$ to be -0.11 and -0.10 for $\mathrm{La}_{0.9} \mathrm{Nd}_{0.1} \mathrm{~F}_{3}$ and $\mathrm{LaF}_{3}$, respectively. This result is in agreement with those obtained in [35] using the isotropic continuum phonon model for orbital singlet ions in low.symmetry crystal field; i.e. the rotational contributions are much smaller than those from the strain. In addition, we determined that the spin-phonon interaction constant $K_{2}$ given by Eq. (8) could be expressed in terms of the magnetoelastic tensor components as follows:

$$
K_{2}=\frac{-\hbar \omega_{\mathrm{D}}^{4}\left(v_{1}^{-5}+2 v_{\mathrm{t}}^{-5}\right)}{21.44 \pi^{2} \rho}\left(G_{11}+G_{12}+G_{13}\right)
$$

\section{7. $\mathrm{Nd}^{3+}$ and $\mathrm{Gd}^{3+}$ spin-lattice relaxation times}

The full linewidth at half peak of a Gaussian distribution due to dipole-dipole and exchange interactions, is given by the expression [36, 37]:

$$
\Delta B_{\mathrm{dip}-\mathrm{ex}}^{2}=\frac{(2.35)^{2} h^{2}\left\langle\Delta \nu^{2}\right\rangle_{\mathrm{av}}}{g^{2} \mu_{\mathrm{B}}^{2}} .
$$

The dipolar interactions between similar ions can be neglected because the distances between $\mathrm{Gd}^{3+}$ ions are sufficiently large in doped crystals. Taking into account dipolar and exchange interactions between dissimilar $\mathrm{Gd}^{3+}$ and $\mathrm{Nd}^{3+}$ ions the following expression is finally obtained for the second moment [38]:

$$
\begin{aligned}
\left\langle\Delta \nu^{2}\right\rangle_{\mathrm{av}} & =\frac{1}{3} S^{\prime}\left(S^{\prime}+1\right) h^{-2} \\
\times & {\left[z J_{\mathrm{p}}^{2}+2 J_{\mathrm{p}} G \sum_{k^{\prime}}^{z}\left(1-3 \gamma_{j k^{\prime}}^{2}\right) r_{j k^{\prime}}^{-3}+G^{2} \sum_{k^{\prime}}^{z}\left(1-3 \gamma_{j k^{\prime}}^{2}\right)^{2} r_{j k^{\prime}}^{-6}\right], }
\end{aligned}
$$

where $G=g g^{\prime} \mu_{\mathrm{B}}^{2} \mu_{0}, h$ is the Planck constant, $g$ and $g^{\prime}$ are $\mathrm{Gd}^{3+}$ and host $\mathrm{Nd}^{3+} g$-factor, respectively, $S^{\prime}\left(=\frac{1}{2}\right)$ is the effective spin of $\mathrm{Nd}^{3+}$ host ion, $\mu_{\mathrm{B}}$ is the Bohr magneton, $\mu_{0}=4 \pi \times 10^{-7} \mathrm{H} \mathrm{m}^{-1}, J_{\mathrm{p}}$ is the average host-impurity pair exchange constant, $z(=1)$ is the number of paramagnetic neighbor $\mathrm{Nd}^{3+}$ ions 
to a $\mathrm{Gd}^{3+}$ ion in $\mathrm{La}_{0.9} \mathrm{Nd}_{0.1} \mathrm{~F}_{3}$ (the remaining neighbors are $\mathrm{La}^{3+}$ ions), $r_{j k^{\prime}}$ are the distances between the $j$ and $k^{\prime}$ ions, and $\gamma_{j k^{\prime}}$ are the direction cosines of $r_{j k^{\prime}}$ with the magnetic field $\boldsymbol{B} \| \boldsymbol{Z}$. The $\mathrm{Gd}^{3+}-\mathrm{Nd}^{3+}$ average pair exchange-interaction constant $J_{\mathrm{p}}\left(\mathcal{H}_{\mathrm{p}}=J_{\mathrm{p}} \boldsymbol{S} \boldsymbol{S}^{\prime}\right)$ is estimated for the paramagnetic host $\mathrm{La}_{0.9} \mathrm{Nd}_{0.1} \mathrm{~F}_{3}$ to be $J_{\mathrm{p}}=-2.7 \pm 0.5 \mathrm{GHz}$ [15]. The effective host $g^{\prime}$ value is determined from the Boltzmann weighting of the $g_{i}$ value for each of the five Kramers doublets in the ${ }^{4} I_{9 / 2}$ ground multiplet of $\mathrm{Nd}^{3+}$ ion in $\mathrm{La}_{0.9} \mathrm{Nd}_{0.1} \mathrm{~F}_{3}$ as follows [39]:

$$
g^{\prime}=\frac{\sum_{i=1}^{5} g_{i} \exp \left(-\Delta_{i} / k T\right)}{\sum_{i=1}^{5} \exp \left(-\Delta_{i} / k T\right)}
$$

where $\Delta_{i}$ is energy of the $i$-th doublet. The $g_{i}$ values are taken from Ref. [9]. From Eq. (15), the effective $g^{\prime}$ values, along the $Z$-axis, have been determined to be $2.40,2.87$, and 3.39 at $4.2,77$, and $295 \mathrm{~K}$, respectively.

The EPR linewidths $\Delta B_{\mathrm{pp}}^{\mathrm{c}}\left(=\Delta B_{\mathrm{dip}-\mathrm{ex}} / 1.18\right)$ of $\mathrm{Gd}^{3+}$ in $\mathrm{La}_{0.9} \mathrm{Nd}_{0.1} \mathrm{~F}_{3}$ crystal for $\boldsymbol{B} \| \boldsymbol{Z}$ are calculated, from Eqs. (13) and (14), to be 370,438 , and $510 \mathrm{mT}$ at $4.2,77$, and $295 \mathrm{~K}$, respectively. The main contribution to these linewidths comes from dipole-dipole interactions whereas contribution from exchange interactions is not larger than $4 \%$. The measured EPR linewidths $\Delta B_{\mathrm{pp}}$ in this crystal equal to $13.8,8.5$, and $8.1 \mathrm{mT}$ at $4.2,77$, and $295 \mathrm{~K}$, respectively, are several times narrower than calculated $\Delta B_{\mathrm{pp}}^{\mathrm{c}}$. This strong and temperature dependent narrowing of the EPR resonance lines can be explained with the mechanism of the spin-lattice modulated narrowing proposed by Mitsuma [40]. Finally, the spin-lattice relaxation time of the host $\mathrm{Nd}^{3+}$ ions can be determined from the expression

$$
\tau_{1}^{\prime}=\frac{3}{20} \frac{h \Delta B_{1 / 2}}{g^{\prime} \mu_{\mathrm{B}} \Delta B_{\mathrm{dip}-\mathrm{ex}}^{2}} .
$$

The EPR lines of $\mathrm{Gd}^{3+}$-doped $\mathrm{La}_{0.9} \mathrm{Nd}_{0.1} \mathrm{~F}_{3}$ (and $\mathrm{LaF}_{3}$ ) crystals have a Lorentzian shape at all temperatures of investigation $\left(\Delta B_{1 / 2}=\sqrt{3} \Delta B_{\mathrm{pp}}\right)$. The superhyperfine interactions of $\mathrm{Gd}^{3+}$ ion with the neighboring ${ }^{19} \mathrm{~F}$ nuclei cause the increase in the linewidth by $1.4 \mathrm{mT}$ [41] which is subtracted from $\Delta B_{\mathrm{pp}}$. Finally, the $\tau_{1}^{\prime}$ 's in $\mathrm{La}_{0.9} \mathrm{Nd}_{0.1} \mathrm{~F}_{3}$ are evaluated to be $(5.02 \pm 0.3) \times 10^{-13},(1.72 \pm 0.3) \times 10^{-13}$, and $(1.01 \pm 0.2) \times 10^{-13} \mathrm{~s}$ at $4.2,77$, and $295 \mathrm{~K}$, respectively. The spin-lattice relaxation times of $\mathrm{Nd}^{3+}$ ions are dependent on the concentration $(x)$ of diamagnetic $\mathrm{La}^{3+}$ ions in mixed crystals $\mathrm{La}_{x} \mathrm{Nd}_{1-x} \mathrm{~F}_{3}$, being longer for bigger concentration. It can be confirmed for $\tau_{1}^{\prime}$ 's at $295 \mathrm{~K}$, because data for the full range of the concentration are available, i.e. $8.87 \times 10^{-15}(x=0), 3.24 \times 10^{-14}(x=0.1)$ and $1.01 \times 10^{-13} \mathrm{~s}$ $(x=0.9)$. The determined $\tau_{1}^{\prime}$ 's at $145 \mathrm{~K}$ are as follows: $3.16 \times 10^{-14}(x=0)$ and $6.30 \times 10^{-14} \mathrm{~s}(x=0.1)$.

The spin-lattice relaxation time of $\mathrm{Gd}^{3+}$ ions $\left(\tau_{1}\right)$ can be determined from the EPR linewidth of $\mathrm{Gd}^{3+}$-doped $\mathrm{LaF}_{3}$ after subtracting the linewidth equal to $1.4 \mathrm{mT}$. The estimated $\tau_{1}$ 's using Heisenberg's uncertainty rule (assuming $h \Delta \nu=$ $\left.g \mu_{\mathrm{B}} \Delta B_{1 / 2}\right)$ are equal to $3.7 \times 10^{-9}, 2.8 \times 10^{-9}$, and $2.5 \times 10^{-9} \mathrm{~s}$ at $4.2,77$, and $295 \mathrm{~K}$, respectively, being longer than $\tau_{1}^{\prime}$ of host $\mathrm{Nd}^{3+}$ ions. It can be assumed that $\tau_{1}$ of $\mathrm{Gd}^{3+}$ ions in $\mathrm{La}_{0.9} \mathrm{Nd}_{0.1} \mathrm{~F}_{3}$ is the same, because the $\mathrm{Gd}^{3+}$ spin-phonon interaction constant $K_{2}$ does not differ within experimental errors from that in $\mathrm{LaF}_{3}\left(\mathrm{Gd}^{3+}\right)$ as shown in Sec. 5 . The $\tau_{1}$ increase by $45 \%$ with lowering temperature 
from 295 to $4.2 \mathrm{~K}$. This rather small increase is due to weak coupling of $\mathrm{Gd}^{3+}$ ions with the lattice, in contrary to strong coupling of $\mathrm{Nd}^{3+}$ ions $\left(\tau_{1}^{\prime}\right.$ increase by $400 \%$ in the same temperature range).

\section{Concluding remarks}

The small local rotations $\left(\Delta \varphi= \pm 3.5^{\circ}\right)$ of the eight fluorine ions (grouped in pairs) surrounding the $\mathrm{Gd}^{3+}$ ion, are determined in the $\mathrm{Gd}^{3+}$-doped $L a_{0.9} \mathrm{Nd}_{0.1} \mathrm{~F}_{3}$ when temperature is lowered down to liquid helium. This can induce a slight distortion of the site symmetry of $\mathrm{Gd}^{3+}$ ion $\left(C_{2 v}\right.$ to $\left.C_{2}\right)$ observed at low temperatures (below $77 \mathrm{~K}$ ).

The energy of single oscillator has been determined to be $302 \mathrm{~cm}^{-1}$ for $\mathrm{La}_{0.9} \mathrm{Nd}_{0.1} \mathrm{~F}_{3}$ and $282 \mathrm{~cm}^{-1}$ for $\mathrm{LaF}_{3}$ using the Einstein model of phonon spectrum. These above average values are somewhat higher than values determined from the Debye model in the long-wavelength limit. The determined Debye temperature $406 \mathrm{~K}$ for $\mathrm{LaF}_{3}$ is close to the realistic value as discussed in Sec. 5 .

The main contribution to the observed EPR linewidths of $\mathrm{Gd}^{3+}$ in $\mathrm{La}_{0.9} \mathrm{Nd}_{0.1} \mathrm{~F}_{3}$ comes from the strong $\mathrm{Nd}^{3+}$ spin-lattice and the $\mathrm{Gd}^{3+}-\mathrm{Nd}^{3+}$ dipoledipole interactions. Contribution from the exchange interaction between $\mathrm{Gd}^{3+}$ and $\mathrm{Nd}^{3+}$ ions plays a secondary role. The $\mathrm{Gd}^{3+}$ spin-phonon interaction constant equal to $0.08 \div 0.13 \mathrm{GHz}$ means that there exist weak interactions of the $\mathrm{Gd}^{3+}$ spin with optical phonons at $302 \mathrm{~cm}^{-1}$, due to the zero orbital momentum for $\mathrm{Gd}^{3+}\left({ }^{8} S_{7 / 2}\right)$. It is also concluded that there is more stronger $\mathrm{Nd}^{3+}$ spin-phonon interaction, i.e. the $\mathrm{Nd}^{3+}$ spin with the optical phonons at $302 \mathrm{~cm}^{-1}$, because the spin-lattice relaxation time $\left(\tau_{1}^{\prime}\right)$ of $\mathrm{Nd}^{3+}$ ions is four orders of magnitude shorter than that of $\mathrm{Gd}^{3+}$ ions $\left(\tau_{1}\right)$.

The sum values of magnetoelastic tensor components $\left(G_{11}+G_{12}+G_{13}\right)$ are calculated for $\mathrm{Gd}^{3+}$ ions to be in the range $-3.33 \div-3.04 \mathrm{GHz}$ for investigated crystals. Further, the values of $\mathcal{R}_{\mathrm{D}}$ in $\mathrm{La}_{0.9} \mathrm{Nd}_{0.1} \mathrm{~F}_{3}$ and $\mathrm{LaF}_{3}$ crystals show that the rotational contributions to the $\mathrm{Gd}^{3+}$ spin-Hamiltonian parameters from phonons are much smaller than those from the strain.

\section{References}

[1] E. Auffray, S. Baccaro, T. Beckers, Y. Benhammou, A.N. Belsky, B. Borgia, D. Boutet, R. Chipaux, I. Dafinei, F. de Notaristefani, P. Depasse, C. Dujardin, H. El Mamouni, J.L. Faure, J. Fay, M. Goyot, S.K. Gupta, A. Gurtu, H. Hillemanns, B. Ille, T. Kirn, M. Lebeau, P. Lebrun, P. Lecoq, J.A. Mares, J. P. Martin, V.V. Mikhailin, B. Moine, J. Nelissen, M. Nikl, C. Pedrini, R. Raghavan, P. Sahuc, D. Schmitz, M. Schneegans, J. Schwenke, S. Tavernier, V. Topa, A.N. Vasil'ev, M. Vivargent, J.P. Walder, Nucl. Instrum. Methods Phys. Res. A 383, 367 (1996).

[2] P. Dorenbos, J.T.M. De Haas, C.W.E. Van Eijk, J. Lumin. 69, 229 (1996).

[3] D. Negoy, T. Purohit, Phys. Status Solidi B 131, 329 (1985).

[4] A.A. Kaminskii, H.R. Verdun, Phys. Status Solidi A 129, K119 (1992).

[5] S.K. Misra, P. Mikołajczak, S. Korczak, J. Chem. Phys. 74, 922 (1981).

[6] S.K. Misra, P. Mikołajczak, N.R. Lewis, Phys. Rev. B. 24, 3729 (1981).

[7] W. Korczak, M.L. Paradowski, L.E. Misiak, Phys. Status Solidi B 165, 203 (1991). 
[8] M.L. Paradowski, W. Korczak, J. Pierre, M. Subotowicz, Phys. Status Solidi B 175, 135 (1993).

[9] M.L. Paradowski, A. Pacyna, A. Bombik, W. Korczak, S.Z. Korczak, J. Magn. Magn. Mater. 166, 231 (1997).

[10] W. Korczak, P. Mikołajczak, J. Cryst. Growth 61, 601 (1983).

[11] M. Mansmann, Z. Kristallogr. 122, 375 (1965).

[12] A. Zalkin, D.H. Templeton, T.E. Hopkins, Inorg. Chem. 5, 1466 (1966).

[13] L. Vegard, Z. Cryst. 67, 239 (1928).

[14] L.E. Misiak, S.K. Misra, P. Mikołajczak, Phys. Rev. B 38, 8673 (1988).

[15] M.L. Paradowski, L.E. Misiak, Nukleonika 42, 543 (1997).

[16] A. Abragam, B. Bleaney, Electron Paramagnetic Resonance of Transition Ions, Clarendon, Oxford 1970.

[17] S. K. Misra, J. Magn. Reson. 23, 403 (1976).

[18] L.E. Misiak, P. Mikołajczak, Acta Phys. Pol. A 75, 621 (1989).

[19] F.J. Owens, C.P. Jr. Poole, H.A. Farach, Magnetic Resonance of Phase Transitions, Academic Press, New York 1979.

[20] D.J. Newman, W. Urban, J. Phys. C 5, 3101 (1972).

[21] D.J. Newman, W. Urban, Adv. Phys. 24, 793 (1975).

[22] K.W. Blazey, T.L. Estle, E. Holzschuh, W. Odermatt, B.D. Patterson, Phys. Rev. B 27, 15 (1983).

[23] K.A. Müller, W. Berlinger, J. Albers, Phys. Rev. B 32, 5837 (1985).

[24] R. Laiho, M. Lakkisto, T. Levola, Philos. Mag. A 47, 235 (1983).

[25] L.E. Misiak, M. Subotowicz, Solid State Commun. 80, 761 (1991).

[26] E. Liarokapis, E. Anastassakis, G.A. Kourouklis, Phys. Rev. B 32, 8346 (1985).

[27] J. Sytsma, W. Van Schaik, G. Blasse, J. Phys. Chem. Solids 52, 419 (1991).

[28] H. Gerlinger, G. Schaack, Phys. Rev. B 33, 7438 (1986).

[29] G.A. Alers, Physical Acoustics: Principles and Method, Vol. 3, Part B, Academic Press, New York 1965.

[30] M.B. Schulz, C.D. Jeffries, Phys. Rev. 149, 270 (1966).

[31] S.B. Oseroff, R. Calvo, Phys. Rev. B 5, 2474 (1972).

[32] N. Guskos, J. Kuriata, T. Rewaj, Acta Phys. Pol. A 81, 659 (1992).

[33] Y.V. Orlowskii, R.J. Reeves, R.C. Powell, T.T. Basiev, K.K. Pukhov, Phys. Rev. $B$ 49, 3821 (1994).

[34] C.A. Bates, H. Szymczak, Phys. Status Solidi B 74, 225 (1976).

[35] C.A. Bates, H. Szymczak, Z. Phys. B 28, 67 (1977).

[36] L.E. Misiak, Acta Phys. Pol. A 83, 195 (1993).

[37] S.K. Misra, U. Orhun, Phys. Rev. B 39, 2856 (1989).

[38] J.H. Van Vleck, Phys. Rev. 74, 1168 (1948).

[39] V.M. Malhotra, J.M. Dixon, H.A. Buckmaster, Physica B 101, 147 (1980).

[40] T. Mitsuma, J. Phys. Soc. Jpn. 17, 128 (1962).

[41] V.K. Sharma, J. Chem. Phys. 54, 496 (1971). 